\title{
Palinotaxonomia de espécies de ACACIa (LEGUMINOSAE-MiMOSOIDEAE) NO SEMI-ÁRIDO BRASILEIRO
}

\author{
Ana Luiza Du Bocage ${ }^{1}$, Mariana Albuquerque de Souza², \\ Silvia Teresinha Sfoggia Miotto ${ }^{3}$ \& Vania Gonçalves-Esteves ${ }^{2,4}$
}

\begin{abstract}
RESUMo
(Palinotaxonomia de espécies do gênero Acacia (Leguminosae-Mimosoideae) no semi-árido brasileiro) Foi realizado o estudo palinológico de 12 espécies de Acacia: A. farnesiana (Acacia subg. Acacia) e A. bahiensis, A. globosa, A. kallunkiae, A. langsdorffii, A. martiusiana, A. monacantha, A. piauhiensis, A. polyphylla, A. riparia, A. tenuifolia e A. velutina (Acacia subg. Aculeiferum). Os grãos de pólen foram acetolisados, medidos, descritos e ilustrados sob microscópio de luz e eletrônico de varredura. Os grãos de pólen em Acacia são reunidos em políades calimadas, médias ou grandes, de contorno esferoidal, em vista frontal e elíptico, em vista lateral, com 16 grãos de pólen, organizados de forma regular, com oito grãos de pólen em cada face, 4-porados. Em A. farnesiana, são observados 24-32 grãos de pólen organizados de forma irregular, 3 -sulcados. Os grãos de pólen da políade são pequenos, subquadrados na face distal e piramidal, quando em vista equatorial. A sexina é granulada ou rugulada, na maioria das espécies. Conclui-se que as espécies não podem ser separadas através das suas características palinológicas, com exceção de A. farnesiana.
\end{abstract}

Palavras-chave: palinologia, semi-árido, caatinga, Mimosaceae, Fabaceae.

\section{Abstract}

(Palynotaxonomy of the species of the Acacia (Leguminosae-Mimosoideae) from the Brasilian semi-arid region) The pollen of 12 species of Acacia: A.farnesiana (L.) Willd. (Acacia subg. Acacia) e A. bahiensis Benth., A. globosa A. Bocage \& S. Miotto, A. kallunkiae Grimes \& Barneby, A. langsdorffii Benth., A. martiusiana (Steud.) Burkart, A. monacantha DC., A. piauhiensis Benth., A. polyphylla DC., A. riparia Kunth, A. tenuifolia (L.) Willd., and A. velutina DC. (Acacia subg. Aculeiferum) was studied. Material was acetolized, measured, described and illustrated using light and scanning electron microscopy. The pollen grains in Acacia are bound in medium or large calimad polyads, spheroidal in shape on frontal view and elliptical from the side, containing 16 pollen grains, organized regularly with 8 pollen grains on each side. Pollen grains are 4-porate. In A. farnesiana, 24-32 pollen grains are found irregularly organized, while pollen grains are 3-sulcate. The polyad pollen grains are small, subsquared on distal view and pyramidal on the equatorial view, sexine is thicker than the nexine. Species could not be separated by their palynological features, except for A. farnesiana. Key words: palynology, semi-arid, caatinga, Mimosaceae, Fabaceae.

\section{INTRODUÇÃO}

O gênero Acacia Mill. compreende 1.350 espécies de distribuição cosmopolita (Maslin et al. 2003). Segundo a classificação de Vassal (1972), este gênero é dividido em três subgêneros, Acacia, Aculeiferum e Phyllodinae. No Brasil são encontradas 44 espécies de Acácia, sendo constatados 16 táxons nativos no semi-árido: A. bahiensis, $A$. farnesiana, A. globosa, A. kallunkiae, A. langsdorffii, A. lewisii, A. limae, A. martiusiana, A. monacantha, A. piauhiensis, A. polyphylla, A. ricoae, A riparia, A. santosii, A. tenuifolia e A. velutina (RicoArce 2003). As espécies de Acacia são caracterizadas pelas folhas bipinadas, geralmente aculeadas, pecíolos com glândulas (nectários extraflorais) e androceu com numerosos estames, brancos, cremes ou amarelos.

Artigo recebido em 12/2007. Aceito para publicação em 05/2008.

${ }^{1}$ Empresa Pernambucana de Pesquisa Agropecuária-IPA. Av. San Martin, 1371, Bongi, 52000-000 Recife, PE, Brasil. ${ }^{2}$ Universidade Federal do Rio de Janeiro, Museu Nacional, Depto. Botânca, São Cristóvão, 20940-040, Rio de Janeiro, RJ, Brasil.

${ }^{3}$ Universidade Federal do Rio Grande do Sul, Departamento de Botânica. Av. Bento Gonçalves 9500, bloco IV, prédio 43433, 91501-970, Porto Alegre, RS, Brasil.

${ }^{4}$ Autor para correspondência: esteves.vr@gmail.com 
Vários estudos palinológicos já foram realizados com as Mimosoideae, sendo o gênero Acácia caracterizado por possuir políades com um número variável de grãos de pólen, podendo conter de quatro a 64 unidades (Wodehouse 1935; Cookson 1953; Coetzee 1955; Barth \& Yoneshigue 1966; Caccavari 1970; Vassal 1972; Robbertse 1974; Guinet \& Vassal 1978; Guinet 1981; Roubik \& Moreno 1991; Carreira et. al. 1996; Silvestre-Capelato \& Melhem 1997; Colinvaux et al. 1999; Caccavari \& Dome 2000; Rico-Arce \& Banks 2001; Moura et al. 2004).

Guinet \& Vassal (1978) levantam hipóteses para diferenciar os grupos dentro do gênero Acacia reconhecendo três principais tipos polínicos, com base na estrutura da exina e na abertura. No tipo 'não especializado' as columelas são muito curtas ou ausentes na sexina e a abertura é do tipo porado. Já no tipo 'especializado' as columelas são conspícuas com abertura do tipo extraporado, enquanto o tipo 'altamente especializado' tem como principal característica a presença de abertura do tipo colporado. A seção Monacanthea, para esses autores, apresenta grãos de pólen do tipo 'não especializado' e a seção Acacia possui dois tipos: 'especializado' e 'altamente especializado'.

Guinet (1986) faz um estudo abrangente das características polínicas em Mimosoideae, concluindo que a morfologia polínica em Acacia é comparável a de Ingeae. Em Acacia subg. Aculeiferum e Acacia subg. Heterophyllum (=Phyllodineae), taxonomicamente próximos, a exina é columelada e as aberturas são poros (simples). A diferença entre eles está na presença de retículo supratectal em Acacia subg. Heterophyllum (=Phyllodineae) que tende a mostrar maior especialização, representada pela ocorrência muito freqüente de pseudoaberturas (extraporos). Em Acacia subg. Acacia, ao contrário, a exina possui estrutura columelar, a abertura é do tipo complexo (colporado), localizada nas partes distais do grão de pólen.
Segundo Guinet (1986), o tipo de abertura e a estrutura da exina parecem ser características mais estáveis dentro de Acacia. Neste gênero, três tipos polínicos têm sido reconhecidos: colpado, colporado e porado.

Caccavari \& Dome (2000), ao estudarem os grãos de pólen de espécies pertencentes à Acacia subg. Aculeiferum, sec. Monacanthea reconhecem a presença de subpseudocolpos em diversos táxons.

Rico-Arce \& Banks (2001) fazem um estudo preliminar de caracteres morfológicos e, entre eles, os polínicos, de espécies neotropicais de Acacia subg. Aculeiferum. Com base em seus resultados, esses autores concluem que o grupo em tela é monofilético e usam com suporte para essa conclusão os seguintes caracteres: polínico - presença de infrateto columelar/ fragmentado e granular; endexina maior que 0,2 mm; macromorfológico - ausência de espinhos e presença de disco no ovário.

Entre os tipos vegetacionais do domínio do semi-árido, a vegetação de caatinga constitui a paisagem dominante (Egler 1951; Andrade Lima 1966; Fernandes \& Bezerra 1990; Souza et al. 1993 e Rodal 1992). Este domínio ocupa uma área de $788.064 \mathrm{~km}^{2}$ e abriga, além da caatinga, outras formações vegetais com fisionomia e flora diferenciadas, como as florestas e os carrascos situados nos enclaves úmidos e subúmidos, e extensas faixas ecotonais com o cerrado e a floresta atlântica (Souza et al 1993).

O presente trabalho visa à caracterização polínica de 12 espécies de Acacia subg. Acacia (A. farnesiana) e Acacia subg. Aculeiferum (os demais táxons) do semi-árido brasileiro, com o objetivo de auxiliar a taxonomia do grupo.

\section{Material e Métodos}

O material analisado foi obtido de botões florais, em sua maioria, coletado no campo e também retirado de exsicatas depositadas nos seguintes herbários: EAC, HRB, HUEFS, ICN, IPA, PEUFR e RB (siglas segundo Holmgren et al. 1990). Dentre as espécies encontradas no semi-árido, Acacia lewisii, A. limae, A. ricoae e $A$. santosii, não tiveram seus grãos de pólen analisados, por falta de material fértil. 
Para cada espécie, sempre que possível, procurou-se examinar três espécimes. Destes, um deles foi escolhido como padrão e indicado no material examinado por um asterisco (*) junto ao número de coleta. Este padrão foi utilizado para mensurações, descrições e ilustrações polínicas enquanto os outros foram utilizados para comparação dos resultados.

A relação do material estudado é fornecida abaixo:

A. bahiensis Benth. - BRASIL. BAHIA: Itiuba, 28.I.2002, J. G. Nascimento 40* (HUEFS); PERNAMBUCO: Belo Jardim, 6.III.2002, A. Bocage 826 (ICN); Caruaru, 28.X.2003, A. Bocage 901 (ICN); BAHIA: Barro Alto, 8.IV.2002, T. S. Nunes 909 (HUEFS).

A. farnesiana (L.) Willd. - BRASIL: ALAGOAS: Piranhas, 3.VII.2000, R. A. Silva 1539*(HRB).

A. globosa A. Bocage \& S. Miotto - BRASIL. BAHIA: Palmeiras, 3.X.2003, A. Bocage 870*(ICN). A. kallunkiae Grimes \& Barneby - BRASIL. BAHIA: rod. 265, trecho que liga a BR415 com Caatiba, 3.III.1978, S. A. Mori et al. 9373* (RB).

A. langsdorffii Benth.- BRASIL. BAHIA: Abaíra, 5.X.2003, A. Bocage 900* (ICN); PIAUÍ: s.d, $E$. Nunes \& P. Martins s.n (EAC 7471). CEARÁ: Aiuaba, s.d, F. A. Viana $s / n$ (EAC 11963). PIAUÍ: Serra da Capivara, 1979, L. Empaire 530 (IPA). A. martiusiana (Steud.) Burkart-BRASIL. BAHIA: Mucugê, Capão, 24.I.2000, L. P. Queiroz 5635* (HUEFS); id:: Mucugê, 31.I.2000, A. Giulietti 1978 (HUEFS; RB); id.: Elísio Medrado, serra da Jibóia, 2.III.2001, L. P. Queiroz 6466 (HUEFS).

A. monacantha DC. - BRASIL. BAHIA: Palmeiras, 4.X.2003, A. Bocage $886^{*}$ (ICN), id., A. Bocage 881 (ICN).

A. piauhiensis Benth.- BRASIL. BAHIA: Seabra, 3.X.2003, A. Bocage 874* (ICN), PERNAMBUCO: Buíque, fazenda Laranjeiras, 5.V.1995, A. P. S. Gomes et al. 14 (PEUFR).

A. polyphylla DC.- BRASIL. BAHIA: Anguera, 29.IV.1999, F. França et al. 272* (HUEFS); Maracás, 26.II.2000, M. M. Silva 297 (HUEFS), PARAÍBA: Itabaiana, 11.III.2002, A. Bocage 857 (ICN), CEARÁ: Crateús, 25.II.2002, A. Bocage 822 (ICN), PERNAMBUCO: Buíque, 31.X.2003, A. Bocage 914 (ICN); Carnaíba, 8.III.2002, A. Bocage 845 (ICN). A. riparia Kunth - BRASIL. BAHIA: Palmeiras, 5.X.2003, A. Bocage 889*.
A. tenuifolia (L.) Willd - BRASIL. PARAÍBA: Ingá, 11.III.2002, A. Bocage 858* (ICN). PERNAMBUCO: Arcoverde, 7.III.2002, A. Bocage 832 (ICN); Caruaru, 6.III.2003, A. Bocage 829 (ICN); BAHIA: Abaíra, após Brejo de Cima, 5.X.2003, A. Bocage 895 (ICN).

A. velutina DC.- BRASIL. BAHIA: Andaraí, estr. Seabra-Andaraí, 31.III.2002, A. M. Giulietti \& R. M. Harley 2078* (HUEFS); Bonfim, H. M. Curran 134: PERNAMBUCO: Marraial, 12.III.1994, A. M. Miranda 1375 (HUEFS).

Para o estudo em microscopia de luz (ML), o material foi acetolisado (Erdtman 1952) com modificações (Melhem et al. 2003). Foram montadas três lâminas permanentes, por espécime estudado, em gelatina, segundo Kisser (1935) apud Erdtman (1952).

Todas as medidas foram realizadas em um prazo máximo de sete dias após a acetólise (Salgado-Labouriau 1973). No material padrão, foram efetuadas 25 medidas dos diâmetros 1 (D1) e 2 (D2) das políades em vista frontal. Tais medidas foram utilizadas para o cálculo da média aritmética $\left(\mathrm{x}^{-}\right)$, desvio padrão da amostra $(s)$, desvio padrão da média $\left(\mathrm{S}_{\mathrm{x}}^{-}\right)$, coeficiente de variabilidade (CV\%) e intervalo de confiança (IC) a $95 \%$. Foram também realizadas 10 medidas dos diâmetros 1 (D1) e 2 (D2) em vista frontal do material usado para comparação dos resultados, dos diâmetros maior (DM) e menor (Dm) das políades em vista lateral, dos diâmetros polar (DP) e equatorial (DE) dos grãos de pólen formadores das políades e da espessura da exina. Tais medidas foram expressas através de média aritmética $\left(\mathrm{x}^{-}\right)$.

Para análise em microscopia eletrônica de varredura (MEV) o material polínico não acetolisado foi colocado sobre fita de carbono dupla-face previamente aderida a suportes próprios e metalizados com uma fina camada de ouro-paládio por cerca de três minutos.

As descrições polínicas seguem a sequiência adotada por Erdtman (1952) e a terminologia adotada para a caracterização dos grãos de pólen, está de acordo com Barth \& Melhem (1988) e Punt et al. (1999). 


\section{Resultados}

As espécies de Acacia (Figs. 1 e 2) estudadas apresentam os grãos de pólen reunidos em políades calimadas, de tamanho médio $(40-50 \mathrm{~mm})$ ou grande $(>50 \mathrm{~mm})$ apenas em A. globosa, A. riparia e $A$. velutina (Tabs. 1,2 ). Possuem contorno esferoidal, em vista frontal, com dois grupos de quatro grãos de pólen, um em cada face e elíptico, em vista lateral, com oito grãos periféricos (Fig. 1a, h, o; Fig. 2k, r), 4-porados, poros de difícil visualização. Em A. farnesiana, são observados 24-32 grãos de pólen organizados de forma irregular (Fig. 1d-e), 3sulcados, sendo os sulcos melhor visualizados em MEV (Fig. 1f-g).

Os grãos de pólen da políade são pequenos (Tab. 1), de forma subquadrada na face distal e piramidal, quando observados em vista equatorial, com região psilada entre os grãos de pólen (Fig. 1c, n, t; Fig. 2g, j, q ).

A sexina é descrita como granulada, quando observada em ML e rugulada, quando observada sob MEV, na maioria das espécies, com exceção de A. bahiensis (Fig. 1c), A. farnesiana (Fig. $1 \mathrm{f}$ ), A. riparia (Fig. 2n) e A. velutina (Fig. 2t) na qual a sexina é considerada rugulada, tanto sob ML, quanto sob MEV. São facilmente observadas sob MEV as perfurações em A. farnesiana (Fig. 1g), A. riparia (Fig. 2n), A. tenuifolia (Fig. 2q) e A. velutina (Fig. 2t).

Em A. polyphylla (Fig. 2j), constata-se a presença, em algumas regiões, de depressões circulares que podem ter sido provocadas por corpos de Ubisch que se desprenderam durante a preparação para a observação sob MEV. Em outras regiões da superfície dessa espécie, no entanto, a sexina é rugulada.

A sexina possui a mesma espessura da nexina em todas as espécies, com exceção de A. bahiensis, A. globosa e A. kallunkiae, onde é sempre mais espessa do que a nexina (Tab. 3). A espessura da exina fica em torno de $2 \mathrm{~mm}$, na maioria das espécies, no entanto, A. kallunkiae e A. langsdorffii apresentam exina espessa (ca. $5 \mathrm{~mm}$ ). Em A. bahiensis, a exina possui ca. de $3,8 \mathrm{~mm}$ de espessura, enquanto que em A. globosa, a exina possui ca. de 1,5 $\mathrm{mm}$ de espessura.

Os materiais de comparação (Tab. 4) apresentam valores dos diâmetros das políades e dos grãos de pólen diferentes, quando comparados com os do respectivo materialpadrão. Essa caracterítica não pode ser utilizada para separar as espécies.

\section{DiscuSSÃo}

As espécies estudadas apresentaram certa homogeneidade em relação à forma da políade, à quantidade e à disposição dos grãos de pólen formadores das políades, bem como à quantidade e ao tipo de abertura (4-porados). Quanto ao tamanho, no entanto, as espécies puderam ser separadas em duas classes, quando são avaliados os limites do intervalo de confiança do diâmetro 1: políades com 38,6$48,3 \mathrm{~mm}$ e políades com $51,1-59 \mathrm{~mm}$; em relação à A. farnesiana (A. subg. Acacia), única espécie que fugiu ao padrão, por que apresentou maior quantidade de grãos de pólen, com abertura do tipo sulco e disposição irregular dos grãos de pólen nas políades.

O tipo de ornamentação da sexina variou, sendo descrita como granulada, quando a análise foi feita sob ML, e rugulada, quando realizada sob MEV, e em quatro espécies das espécies descrita como rugulada tanto sob ML, quanto sob MEV.

Barth \& Yoneshigue (1966) descreveram, palinologicamente, espécies de Leguminosae (Mimosoideae) ocorrentes em Santa Catarina e, dentre elas, Acacia adhaerens Benth. (atualmente A. martiusiana), A. catharinensis Burkart e A. nitidifolia Speg. Os resultados encontrados pelas autoras, com relação ao tamanho das políades e à organização dos grãos de pólen, foram semelhantes no presente estudo, embora apenas a espécie $A$. martiusiana tenha sido analisada. Foram observadas diferenças no que se refere à ornamentação da sexina (psilada ou variadamente ondulada), enquanto, aqui, a sexina foi descrita como rugulada ou granulada. Essa 

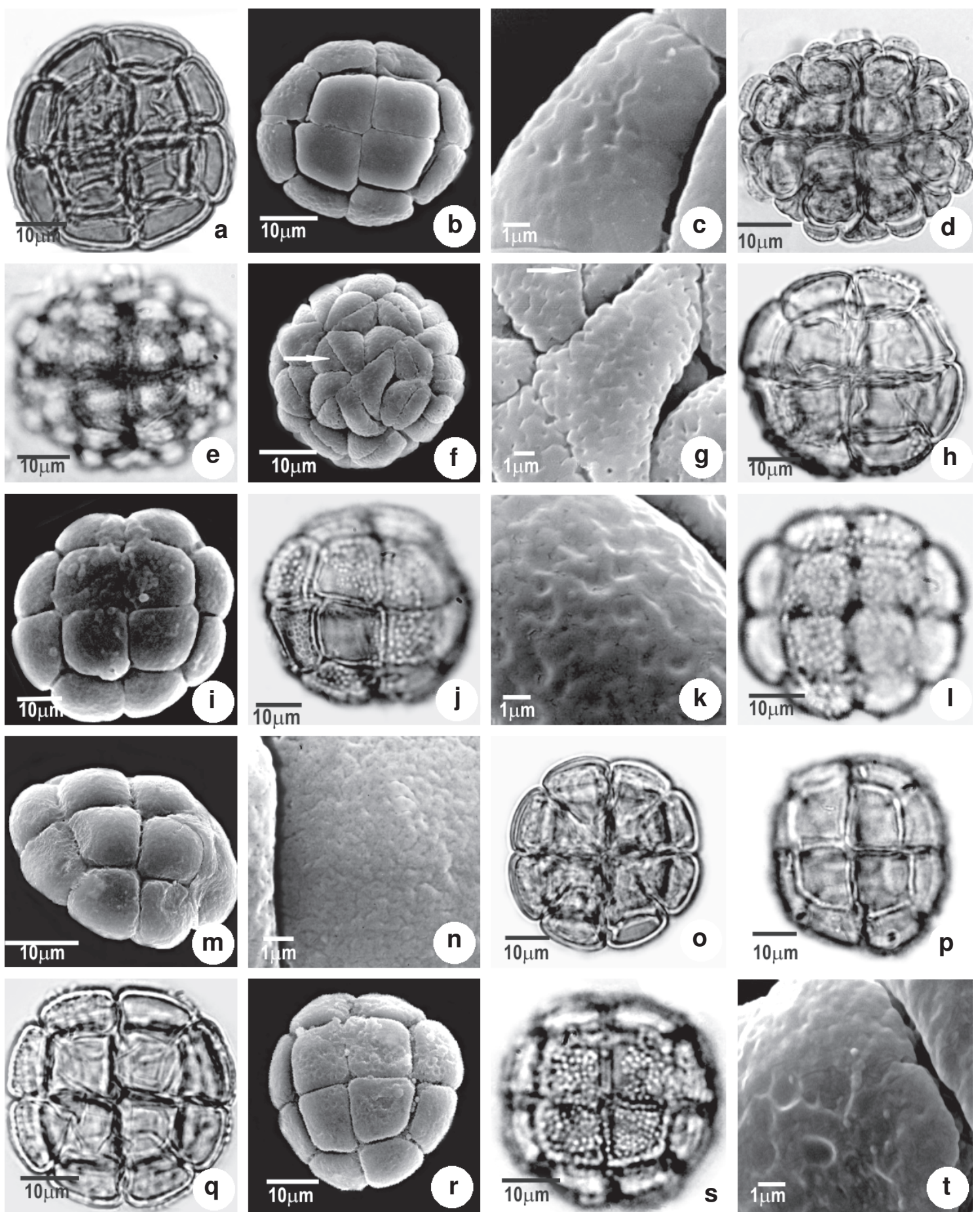

Figura 1 - Fotos e eletromicrografias de espécies de Acacia. a-c. A. bahiensis - vista geral: a. corte óptico; b. superfície; c. detalhe da superfície. d-g. A. farnesiana - vista geral: d. corte óptico; e, f. superfície (seta em f indica o sulco); g. detalhe da superfície (seta indica o sulco). h-k. A. globosa - vista geral: h. corte óptico; i-j. superfície; k. detalhe da superfície. 1-n. A. kallunkiae - vista geral: 1. corte óptico; m. vista lateral, superfície; n. detalhe da superfície. o-p. A. langsdorffii - vista geral: o. corte óptico; p. superfície. q-t. A. martiusiana - vista geral: q. corte óptico; r-s. superfície; t. detalhe da superfície. 

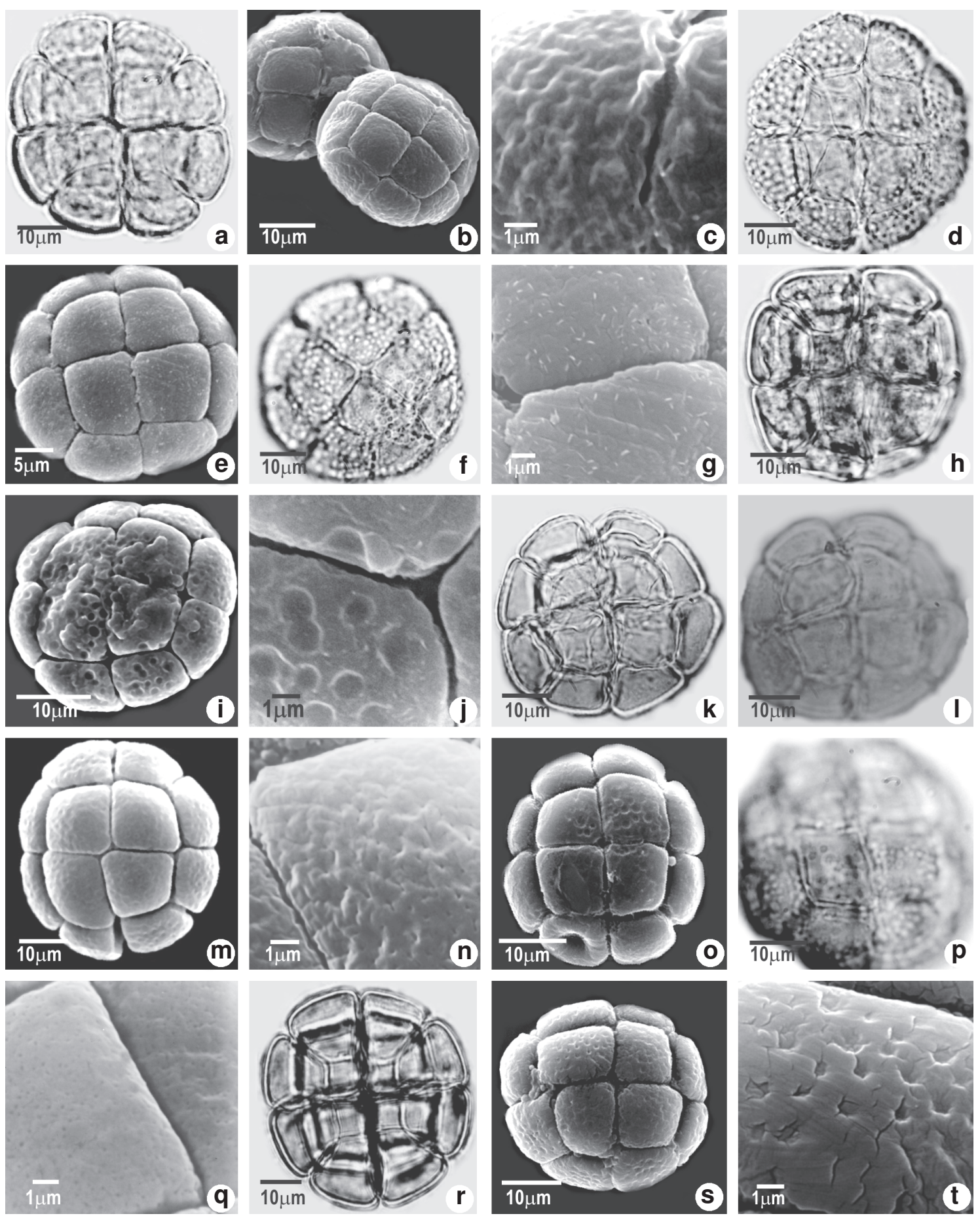

Figura 2 - Fotos e eletromicrografias de espécies de Acacia. a-c. A. monacantha - vista geral: a. corte óptico; b. superfície; c. detalhe da superfície. d-g. A. piauhiensis - vista geral: d. corte óptico; e-f. superfície; g. detalhe da superfície. h-j. A. polyphylla - vista geral: h. corte óptico; i. superfície; j. detalhe da superfície. k-n. A. riparia - vista geral: k. corte óptico; 1-m. superfície; n. detalhe da superfície. o-q. A. tenuifolia - vista geral: o-p. superfície; q. detalhe da superfície. r-t. A. velutina - vista geral: r. corte óptico; s. superfície; t. detalhe da superfície. 
Tabela 1 - Diâmetros (em $\mu \mathrm{m})$ das políades de espécies de Acacia. média \pm desvio padrão $\left(\mathrm{x}^{-} \pm \mathrm{s}_{\mathrm{x}^{-}}\right)$ $(n=25)$. São fornecidos a amplitude dos dados e o intervalo de confiança (IC) a $95 \%$.

\begin{tabular}{lcccccc}
\hline & \multicolumn{3}{c}{ Diâmetro 1 } & \multicolumn{3}{c}{ Diâmetro 2 } \\
\hline Espécies & $\begin{array}{c}\text { Faixa de } \\
\text { Variação }\end{array}$ & $\mathbf{x}^{-} \pm \mathbf{s}_{\mathbf{x}} \mathbf{x}^{-}$ & IC 95\% & $\begin{array}{c}\text { Faixa de } \\
\text { Variação }\end{array}$ & ${\mathbf{x} \pm \mathbf{x}^{-}}^{-}$ & IC 95\% \\
\hline A. bahiensis & $40,0-47,5$ & $45,2 \pm 0,4$ & $44,4-46,0$ & $37,5-47,5$ & $42,5 \pm 0,5$ & $41,5-43,5$ \\
A. farnesiana & $45,0-50,0$ & $47,3 \pm 0,5$ & $46,7-48,3$ & $42,5-47,5$ & $45,1 \pm 0,5$ & $44,1-46,1$ \\
A. globosa & $50,0-62,5$ & $55,0 \pm 0,8$ & $53,4-56,6$ & $47,5-52,5$ & $50,2 \pm 0,3$ & $49,6-50,8$ \\
A. kallunkiae & $37,5-42,5$ & $40,2 \pm 0,4$ & $39,4-41,0$ & $35,0-42,5$ & $39,1 \pm 0,5$ & $38,1-40,1$ \\
A. langsdorffii & $42,5-50,0$ & $46,1 \pm 0,5$ & $45,1-47,1$ & $40,0-50,0$ & $44,7 \pm 0,6$ & $43,5-45,9$ \\
A. martiusiana & $37,5-42,5$ & $40,0 \pm 0,3$ & $39,4-40,6$ & $37,5-42,5$ & $38,3 \pm 0,3$ & $37,7-38,9$ \\
A. monacantha & $42,5-47,5$ & $45,0 \pm 0,5$ & $44,0-46,0$ & $40,0-47,5$ & $44,9 \pm 0,4$ & $44,1-45,7$ \\
A. piauhiensis & $42,5-47,5$ & $45,0 \pm 0,2$ & $44,6-45,4$ & $40,0-45,0$ & $42,0 \pm 0,4$ & $41,2-42,8$ \\
A. polyphylla & $37,5-40,0$ & $39,0 \pm 0,2$ & $38,6-39,4$ & $37,5-40,0$ & $38,3 \pm 0,2$ & $37,9-38,7$ \\
A. riparia & $52,5-60,0$ & $58,0 \pm 0,5$ & $57,0-59,0$ & $50,0-57,5$ & $53,0 \pm 0,5$ & $52,0-54,0$ \\
A. tenuifolia & $40,0-45,0$ & $42,2 \pm 0,3$ & $41,6-42,8$ & $37,5-42,5$ & $39,5 \pm 0,5$ & $38,5-40,5$ \\
A. velutina & $47,5-55,0$ & $52,1 \pm 0,5$ & $51,1-53,1$ & $47,5-57,5$ & $51,0 \pm 0,4$ & $50,2-51,8$ \\
\hline
\end{tabular}

$\mathrm{x}$ - média aritmética; $\mathrm{s}_{\mathrm{x}}$ - desvio padrão da média; IC - intervalo de confiança

diferença talvez esteja relacionada com os recursos aqui utilizados para a observação (MEV).

Caccavari (1970) estudou os grãos de pólen de 19 táxons de Acacieae da Argentina e, dentre eles, três foram aqui analisados: $A$. monacantha, A. polyphylla e A. velutina. As diferenças encontradas nas descrições dessas espécies estão relacionadas ao número de aberturas (as políades podem ter de 4 a 6 poros localizados 4 nos vértices do equador, e 1 ou 2 , no pólo proximal) e à ornamentação da sexina: reticulada em $A$. monacantha, lisa ou levemente ondulada em A. polyphylla. Caccavari (1970) descreveu a sexina de $A$. velutina var. glabrescens como sendo reticulada, não encontrada em $A$. velutina, analisada no presente trabalho.

Guinet (1981), ao analisar as características polínicas nas Mimosoideae, definiu que o teto é liso em Acacia subg. Aculeiferum ou reticulado (retículo supratectal) em Acacia subg. Heterophyllum (=Phyllodineae), mas nos dois subgêneros, a estrutura da exina é columelar. No presente estudo, os resultados são diferentes, pois as espécies de Acacia subg. Aculeiferum não apresentaram teto liso embora, o infrateto também seja columelar.
Guinet (1986) verificou que em Acacia subg. Aculeiferum, as políades possuíam de oito a 16 grãos de pólen, quantidade essa notavelmente menor do que a encontrada em outros subgêneros. Segundo o autor, em Acacia subg. Aculeiferum, a exina é lisa e falta um pseudocolpo bem diferenciado, diferente do que ocorre em Acacia subg. Phyllodineae, no qual a exina é frequentemente suprarreticulada. As espécies de Acacia subg. Aculeiferum, aqui analisadas, diferem dos resultados de Guinet (1986), pois não foi encontrado pseudocolpo.

Silvestre-Capelato \& Melhem (1997) estudaram os grãos de pólen de duas espécies de Acacia: A. grandistipula Benth. e A. paniculata Willd.(atualmente, A. tenuifolia). Para as autoras, A. tenuifolia apresenta políades com exina escabrada. Os resultados, aqui encontrados para a espécie, diferem dos de Silvestre-Capelato \& Melhem (1997), no que se refere à políade (calimada) e à ornamentação da exina (granulada em ML e rugulada em MEV).

Caccavari \& Dome (2000) analisaram os grãos de pólen de 36 táxons de Acacia, (nove aqui também analisadas), utilizando recursos de microscópio de luz e eletrônico de varredura 
Tabela 2 - Diâmetros das políades de espécies de Acacia. Diâmetros (em $\mu \mathrm{m})$ das políades, em vista lateral e dos grãos de pólen em vista frontal, de espécies de Acacia. Dados são média aritmética $(\mathrm{n}=10)$. DM- diâmetro maior; Dm- diâmetro menor em vista lateral; D1 e D2- diâmetros em vista frontal.

\begin{tabular}{lcccc}
\hline Espécies & DM & Dm & D1 & D2 \\
\hline A. bahiensis & 47,7 & 30,5 & 12,5 & 14,4 \\
A. farnesiana & 49,5 & 45,5 & - & - \\
A. globosa & - & - & - & - \\
A. kallunkiae & - & - & 10,0 & 12,5 \\
A. langsdorffii & 45,0 & 32,5 & 15,0 & 14,3 \\
A. martiusiana & 45,0 & 33,0 & 12,5 & 15,0 \\
A. monacantha & 43,0 & 36,3 & 12,0 & 12,5 \\
A. piauhiensis & 45,3 & 29,0 & 12,5 & 12,5 \\
A. polyphylla & 40,0 & 27,0 & 11,5 & 11,5 \\
A. riparia & - & - & - & - \\
A. tenuifolia & - & - & - & - \\
A. velutina & 53,6 & 40,0 & 15,7 & 17,4 \\
\hline
\end{tabular}

e transmissão. Para estas autoras, as espécies se caracterizaram por apresentar políades com 16 grãos de pólen porados, com ornamentação lisa a levemente suprarreticulada ou supramicrorreticulada, com lumens circulares cujos diâmetros correspondem aos corpos de Ubisch; camada infratectal granular e camada basal ausente ou vestigial. Algumas espécies da seção Monacanthea, segundo as autoras, possuem um subpseudocolpo facilmente discernível ou difuso. No presente estudo, não foram observados pseudocolpos, nem ornamentação do tipo descrito. Em uma única espécie foram observados sulcos ( $A$. farnesiana) e não pseudosulcos; nas demais, foram descritos poros. Com relação ao tipo de ornamentação, registrou-se superfície granulada, rugulada ou com perfurações, mas em nenhuma das espécies foram observados retículos ou microrretículos. Talvez a diferença encontrada pelas autoras esteja relacionada à presença das marcas deixadas pelos orbículos.

Rico-Arce \& Banks (2001) estudaram sete espécies de Acacia e, dentre elas, A. velutina descrita como possuindo, principalmente, políades com 16 grãos de pólen, porados, teto
Tabela 3 - Espessura (em mm) das camadas da exina dos grãos de pólen de espécies de Acacia. Dados são média aritmética $(\mathrm{n}=10)$

\begin{tabular}{lccc}
\hline Espécies & Exina & Sexina & Nexina \\
\hline A.bahiensis & 3,8 & 2,5 & 1,3 \\
A. farnesiana & 2,0 & 1,0 & 1,0 \\
A. globosa & 1,5 & 1,0 & 0,5 \\
A. kallunkiae & 5,0 & 2,9 & 2,1 \\
A. langsdorffii & 4,6 & 2,3 & 2,3 \\
A. martiusiana & 2,0 & 1,0 & 1,0 \\
A. monacantha & 2,0 & 1,0 & 1,0 \\
A. piauhiensis & 2,0 & 1,0 & 1,0 \\
A. polyphylla & 2,0 & 1,0 & 1,0 \\
A. riparia & 2,0 & 1,0 & 1,0 \\
A. tenuifolia & 2,0 & 1,0 & 1,0 \\
A. velutina & 2,0 & 1,0 & 1,0 \\
\hline
\end{tabular}

Tabela 4 - Diâmetro (em mm) das políades de espécies de Acacia. Dados são média aritmética (n=10). D1 e D2 são diâmetros em vista frontal.

\begin{tabular}{lll}
\hline Espécies & $\begin{array}{l}\text { D1 } \\
\mathbf{x}\end{array}$ & $\begin{array}{l}\text { D2 } \\
\mathbf{x}\end{array}$ \\
\hline A. bahiensis & & \\
Bocage 826 & 47,3 & 42,0 \\
Bocage 901 & 46,1 & 44,4 \\
Nunes 909 & 45,5 & 42,0 \\
A. langsdorffii & & \\
Empaire 530 & 42,5 & 42,5 \\
Nunes s.n. & 43,5 & 42,8 \\
Viana s.n. & 47,0 & 44,8 \\
A. martiusiana & & \\
Queiroz 6466 & 43,6 & 41,0 \\
Giulietti 1978 & 42,3 & 40,0 \\
A. monacantha & & \\
Bocage 881 & 39,0 & 38,0 \\
A. piauhiensis & & \\
Gomes et al.14 & 55,0 & 47,3 \\
A. polyphylla & & \\
Silva 297 & 40,5 & 38,5 \\
Bocage 882 & 38,8 & 38,3 \\
Bocage 914 & 39,8 & 38,0 \\
Bocage 485 & 39,5 & 39,0 \\
A. tenuifolia & & \\
Bocage 829 & 37,1 & 34,9 \\
Bocage 832 & 39,0 & 37,3 \\
Bocage 895 & 49,0 & 47,0 \\
A. velutina & & \\
Miranda 1375 & 54,6 & 51,7 \\
Curran 134 & 50,3 & 49,5 \\
\hline
\end{tabular}


finamente rugulado ou fracamente crenulado. Com relação à $A$. velutina, os resultados do presente estudo são semelhantes aos encontrados por Rico-Arce \& Banks (2001).

Silva (1990), estudando as espécies do gênero Acacia, ocorrentes na Amazônia brasileira, confeccionou uma chave polínica baseada no número de grãos de pólen e ornamentação da superfície. $\mathrm{O}$ autor caracterizou A. farnesiana como tendo políades com 32 grãos de pólen e $A$. polyphylla com 16 grãos de pólen e sexina psilada. Os resultados, aqui encontrados, diferem de Silva (1990) apenas no que se refere à ornamentação da última espécie. Moura et al. (2004) também analisaram a morfologia polínica de Acacia polyphylla, além de outras espécies de várzea e de igapó da Amazônia Central. Para os autores, a espécie foi descrita como possuindo políades médias, discoidais, calimadas, e exina psilada. Os estudos, aqui apresentados, diferem daqueles encontrados pelos autores, apenas no que se refere à ornamentação da exina (granulada em ML e rugulada em MEV).

Pode-se concluir que as espécies analisadas são um pouco semelhantes em suas características palinológicas, exceto $A$. farnesiana (única espécie do estudo, pertencente a Acacia subg. Acacia, que apresentou políades muito diferentes). No entanto, diferenças importantes podem ser consideradas tais como: tamanho das políades, ornamentação da sexina sob microscópio de luz e espessura da exina. Com base nos resultados, considera-se que o grupo possui certa homogeneidade polínica, sendo $A$. farnesiana a espécie morfopalinologicamente distinta, tanto pelo número de grãos de pólen, quanto pelo tipo de abertura.

\section{Agradecimentos}

Ao Laboratório de Ultraestrutura Celular, do Instituto de Biofísica da Universidade Federal do Rio de Janeiro (UFRJ), na pessoa da técnica de microscopia eletrônica de varredura, Nôemia Rodrigues Gonçalves. Ao CNPq pela bolsa concedida à primeira autora.

\section{REFERÊNCIAS BIBLIOGRÁFICAS}

Andrade Lima, D. 1966. Vegetação. In: IBGE. Atlas Nacional do Brasil. Conselho Nacional de Geografia, Rio de Janeiro, 50p.

Barroso, G. M.; Peixoto, A. L.; Ichaso, C. L. F.; Guimarães, E. F. \& Costa, C. G. 1986. Sistemática de Angiospermas do Brasil. Impr. Univ. Viçosa, Viçosa, 377p.

Barth, O. M. \& Yoneshigue, Y. 1966. Catálogo sistemático dos pólens das plantas arbóreas do Brasil Meridional VIII Leguminosae (Mimosoideae). Memórias do Instituto Oswaldo Cruz 64: 79-123. \& Melhem, T. S. 1988. Glossário Ilustrado de Palinologia. Ed. Unicamp, Campinas.

Caccavari, M. A. 1970. Granos de polen de Leguminosas de la Argentina I. Subfam. Mimosoideae; Tribu Acacieae. Darwiniana 16(1-2): 144-183.

\& Dome, E. A. 2000. Subpseudocolpi in polyads of Acacia, subgenus Aculeiferum. Grana 39: 32-38.

Carreira, L. M. M.; Lopes, J. R. C.; Silva, M. F. \& Nascimento, L. A. S. 1996. Catálogo de pólen das leguminosas da Amazônia brasileira. Museu Paraense Emílio Goeldi, Coleção Adolpho Ducke, Belém, 137p.

Coetzee, J. A. 1955. The morphology of Acacia pollen. South African Journal of Science 52: 23-27.

Cookson, J. 1953. The Caenozoic occurrence of Acacia in Australia. Australian Journal of Botany 2: 52-59.

Colinvaux, P.; De Oliveira, P. E. \& Patino, J. E. M. 1999. Amazon pollen manual and atlas. Harwood Academic Publishers, The Netherlands, 332p.

Egler, W. A. 1951. Contribuição ao estudo da caatinga pernambucana. Revista Brasileira de Geografia 3: 65-77.

Erdtman, G. 1952. Pollen morphology and plant taxonomy - Angiosperms. Almqvisit \& Wiksel, Stockholm, 539p.

Fernandes, A. G. \& Bezerra, P. 1990. Estudo fitogrográfico do Brasil. Editora Styllus Comunicações, Fortaleza. 
Guinet, P. 1981. Mimosoideae: the characters of their pollen grains. In: Polhill, R. M. \& Raven, P. H. (eds). Advances in Legume Systematics. Part. 2.Royal Botanic Gardens, Kew. Pp. 835-857.

1986. Geographic patterns of the main pollen characters in genus Acacia (Leguminosae), with particular reference to subgnus Phyllodineae. In: Pollen and Spores: form and functions (S. Blackmore and I. K. Ferguson, eds.). Academic Press, London. Pp. 297-311.

Guinet, P. \& Vassal, J. 1978. Hypotheses on the differentiation of the major groups in the genus Acacia (Leguminosae). Kew Bulletin 32: 509-527.

Holmgren, P. K.; Holmgren, N. H. \& Bainett, L. G. 1990. Index Herbariorum. Part 1: The Herbaria of the world. $8^{\mathrm{a}}$ ed., New York Botanical Garden, New York.

Maslin, B. R.; Miller, J. T. \& Seigler, D. S. 2003. Overview of the generic status of Acacia (Leguminosae:Mimosoideae). Australian Systematic Botany 16: 1-18.

Melhem, T. S.; Cruz-Barros, M. A. V.; Corrêa, A. M. S.; Makino-Watanabe, H.; Silvestre-Capelato \& Esteves, V. L. G. 2003. Morfologia polínica em plantas de Campos do Jordão (São Paulo, Brasil). Boletim do Instituto de Botânica 16: 1-104.

Moura, C. O.; Absy, M. L.; Santos, F. A. R. \& Marques-Souza, A. C. 2004. Morfologia polínica de espécies de várzea e de igapó da Amazônia Central. Acta Amazonica 34(1): 15-19.

Punt, W.; Blackmore, S.; Nilsson, S. \& Thomas, A. 1999. Glossary of pollen and spore Terminology. http://www.biol.ruu.nl./ $\sim$ palaeo/glossary/glos-int.htm. (capturado em 1999).

Rico-Arce, L. 2003. Geographical patterns in neotropical Acacia (Leguminosae: Mimosoideae). Australian Systematics Botany 16: 41-48.

\& Banks, H. 2001. A preliminary survey of pollen and other morphological characters in neotropical Acacia subgenus Aculeiferum (Leguminosae: Mimosoideae). Botanical Journal of the Linnean Society 135: 263-270.

Robbertse, P. J. 1974. A scanning electron microscopic investigation of the pollen of south African Acacia species. Journal South African Botanic 40: 91-99.

Rodal, M. J. N.; Sampaio, E. V. S. B. \& Figueiredo, M. A. 1992. Manual sobre métodos de estudo florístico e fitossociológico: ecossistema caatinga. Sociedade Botânica do Brasil, São Paulo, 24p.

Roubik, D. W. \& Moreno, J. E. P. 1991. Pollen and spores of Barro Colorado Island. Missouri Botanical Garden, St. Louis,258p.

Salgado-Labouriau, M.L.1973. Contribuição à palinologia dos cerrados. Academia Brasileira de Ciências, Rio de Janeiro, 291p.

Silva, A. S. L. 1990. Taxonomia das espécies do gênero Acacia Mill. ocorrentes na Amazônia brasileira. Boletim Museu Paranaense Emílio Goeldi 6(2): 152-226.

Silvestre-Capelato, M. S. F. \& Melhem, T. S. 1997. Flora polínica da Reserva do Parque Estadual das Fontes do Ipiranga (São Paulo, Brasil). Família: 81 - Leguminosae. Hoehnea 24(1): 115-163.

Souza, M. J. N.; Martins, M. L. R.; Soares, Z. M. L.; Freitas-Filho, M. R.; Pinheiro, F. S. A.; Sampaio, M. A. B.; Carvalho, G. M. B. S.; Soares, A. M. L.; Gomes, E. C. B. \& Silva, R. A. 1993. Redimensionamento da região do semi-árido do Nordeste do Brasil. In: Conferência Nacional e Seminário Latino-Americano de Desertificação. Fundação Esquel do Brasil, Fortaleza, 25p.

Vassal, J. 1972. Apport des recherches ontogeniques et seminologiques a l'étude morphologique, taxonomique et phylogenique du genre Acacia. Bulletin de la societè d'Histoire Naturelle de Toulouse 108: 105-247.

Wodehouse, R. P. 1935. Pollen grains. MacGrawHill Book Co., New York, 574p. 